ELECTRONIC RESEARCH ANNOUNCEMENTS OF THE AMERICAN MATHEMATICAL SOCIETY

Volume 9, Pages 69-79 (September 2, 2003)

S $1079-6762(03) 00113-6$

\title{
METRIC TENSOR ESTIMATES, GEOMETRIC CONVERGENCE, AND INVERSE BOUNDARY PROBLEMS
}

\author{
MICHAEL ANDERSON, ATSUSHI KATSUDA, YAROSLAV KURYLEV, MATTI LASSAS, \\ AND MICHAEL TAYLOR
}

(Communicated by Tobias Colding)

\begin{abstract}
Three themes are treated in the results announced here. The first is the regularity of a metric tensor, on a manifold with boundary, on which there are given Ricci curvature bounds, on the manifold and its boundary, and a Lipschitz bound on the mean curvature of the boundary. The second is the geometric convergence of a (sub)sequence of manifolds with boundary with such geometrical bounds and also an upper bound on the diameter and a lower bound on injectivity and boundary injectivity radius, making use of the first part. The third theme involves the uniqueness and conditional stability of an inverse problem proposed by Gel'fand, making essential use of the results of the first two parts.
\end{abstract}

\section{INTRODUCTION}

Here we announce results on regularity, up to the boundary, of the metric tensor of a Riemannian manifold with boundary, under Ricci curvature bounds and control of the boundary's mean curvature; an application to results on Gromov compactness and geometric convergence in the category of manifolds with boundary; and then an application of these results to the study of an inverse boundary spectral problem introduced by I. Gel'fand. Details are given in AK2LT.

Regularity of the metric tensor away from the boundary has been studied and used in a number of papers, starting with [DTK]. One constructs local harmonic coordinates and uses the fact that, in such harmonic coordinates, the Ricci tensor has the form

$$
\Delta g_{\ell m}-B_{\ell m}(g, \nabla g)=-2 \text { Ric }_{\ell m} .
$$

Here $\Delta$ is the Laplace-Beltrami operator, applied componentwise to the components of the metric tensor, and $B_{\ell m}$ is a quadratic form in $\nabla g_{i j}$, with coefficients that are smooth functions of $g_{i j}$ as long as the metric tensor satisfies a bound $C_{1}|\eta|^{2} \leq$ $g_{j k}(x) \eta^{j} \eta^{k} \leq C_{2}|\eta|^{2}$, with $0<C_{1} \leq C_{2}<\infty$. If one is given information on the

Received by the editors December 17, 2002.

2000 Mathematics Subject Classification. Primary 35J25, 47A52, 53C21.

Key words and phrases. Ricci tensor, harmonic coordinates, geometric convergence, inverse problems, conditional stability.

(C)2003 American Mathematical Society 
Ricci tensor, one can regard (1.1) as an elliptic PDE for the metric tensor, and obtain information on its components, in harmonic coordinates.

The notion of compactness of a family of Riemannian manifolds and of geometric convergence issues from work of J. Cheeger [Ch] and M. Gromov (cf. [Gr], the revised and translated version of his 1981 work). The role of harmonic coordinates in the study of such geometric convergence has been exploited in a number of papers. The paper An1 established compactness given a sup norm bound on the Ricci tensor, an upper bound on the diameter, and a lower bound on the injectivity radius, for a family of compact Riemannian manifolds of a fixed dimension. Convergence was shown to hold, for a subsequence, in the $C^{r}$-topology, for any $r<2$.

One of our motivations to extend the scope of these results to the category of manifolds with boundary arises in the study of a class of inverse problems. In these problems, one wants to determine the coefficients of some partial differential equation in a bounded region via measurements of solutions to the PDE at the boundary. Such problems arise in various areas, including geophysics, medical imaging, and nondestructive testing. One problem, formulated by I. Gel'fand [Ge], consists in finding the shape of a compact manifold $\bar{M}$ with boundary $\partial M$ and the metric tensor on it from the spectral data on $\partial M$. Namely, if $R_{\lambda}$ is the resolvent of the Neumann Laplacian $\Delta^{N}$ on $M$, the Gel'fand data consist of the restriction of the integral kernel $R_{\lambda}(x, y)$ of the resolvent to $x, y \in \partial M$, as $\lambda$ varies over the complement of the real axis. Another formulation of Gel'fand's inverse problem will be given in $\S 4$.

For such an inverse problem, the first issue to investigate is uniqueness. In the context of $C^{\infty}$ metric tensors, this was established for the Gel'fand problem in [BK1]. As we will explain below, it is important to obtain uniqueness with much less regular coefficients.

Once uniqueness results have been obtained, one has to face up to the issue of ill-posedness of the inverse problem. That is, one can make large changes in $\bar{M}$ that have only small effects on boundary data obtained from examining the boundary behavior of the resolvent kernel mentioned above. For example, given $(\bar{M}, g)$, one could take an auxiliary manifold $X$, without boundary, of the same dimension as $\bar{M}$, remove a small ball from $X$ and from the interior of $\bar{M}$, and connect these manifolds by a thin tube. One is faced with the task of stabilizing this ill-posed inverse problem. One ingredient in this process involves having some a priori knowledge of the quantities one is trying to determine, typically expressed in terms of a priori bounds on these quantities in certain norms.

In the case of trying to determine an unknown Riemannian manifold with boundary $\bar{M}$, from boundary spectral data, it is natural to make a priori hypotheses on geometrical properties of $\bar{M}$. Furthermore, if one must make such a priori hypotheses, it is desirable to get by with as weak a set of hypotheses as possible. There is then a tension between the desire to make weak a priori hypotheses and the need to establish uniqueness results. For preliminary results in this direction see $[\mathrm{K} 2 \mathrm{~L}$, [Ka].

In our work we impose a priori sup norm bounds on the Ricci tensor of $\bar{M}$, and of $\partial M$. This, together with a Lipschitz norm bound on the mean curvature of 
$\partial M \hookrightarrow \bar{M}$, is shown to imply certain regularity, up to the boundary, of the metric tensor of $\bar{M}$, when one is in "boundary harmonic coordinates". To be precise, we obtain regularity in the Zygmund space $C_{*}^{2}(\bar{M})$, a degree of regularity being better than $C^{r}$ for any $r<2$ and just slightly worse than $C^{2}$. This result has the following important advantage over a $C^{2-\varepsilon}$ estimate. The Hamiltonian vector field associated with the metric tensor has components with a log-Lipschitz modulus of continuity. Hence, by Osgood's theorem, it generates a uniquely defined geodesic flow, on the interior of $\bar{M}$, and also for geodesics issuing transversally from $\partial M$. A more complete description of these results is given in $\S 2$.

In $\S 3$ we describe a compactness result for families of compact Riemannian manifolds, of dimension $n$, with boundary, for which there are fixed bounds on the sup norms of $\operatorname{Ric}_{M}$ and $\operatorname{Ric}_{\partial M}$, on the Lipschitz norm of the mean curvature of $\partial M$, and on the diameter, and fixed lower bounds on the injectivity and boundary injectivity radius. It turns out that a sequence of such Riemannian manifolds has a subsequence, converging in the $C^{r}$-topology, for all $r<2$, whose limit $(\bar{M}, g)$ has metric tensor in $C_{*}^{2}(\bar{M})$.

In $\S 4$ we discuss Gel'fand's inverse boundary problem, recast in the form of an inverse boundary spectral problem. We describe how, having boundary spectral data, we can recognize whether a given function $h \in C(\partial M)$ has the form $h(z)=$ $r_{x}(z)=\operatorname{dist}(x, z)$, for some $x \in \bar{M}$, all $z \in \partial M$, thus recovering the image in $C(\bar{M})$ of $\bar{M}$ under the boundary distance representation. Such a representation, whose use was initiated in $[\mathrm{Ku}]$ and $\mathrm{KuL}$, plays an important role in the uniqueness proof, but for it to work we need to know that geodesics from points in $\partial M$, pointing normal to the boundary, are uniquely defined. As noted above, this holds when the metric tensor is in $C_{*}^{2}(\bar{M})$, and we obtain a uniqueness result in this category. This fits in perfectly with the compactness result described in $\S 3$, to yield a result on stabilization of this inverse problem.

\section{ACKNOWLEDGMENT}

We thank R. Mazzeo for useful discussions, particularly regarding material in $\S 2$. Also, we thank T. Sakai, T. Sunada, and Y.D. Burago for discussions on the role of geometric convergence, and M. Gromov, G. Uhlmann, and E. Somersalo for interest and support. M. Anderson's work was partially supported by NSF grant DMS-0072591. A. Katsuda's work was partially supported by a JSPS Grant in Aid for Scientific Research (C)(2) No. 14540081. Y. Kurylev and M. Lassas were partially supported by the Royal Society. Also, M. Lassas's work was partially funded by the Academy of Finland. M. Taylor's work was partially supported by NSF grant DMS-0139726. Part of this work was done at MSRI and at the Oberwolfach RiP Program, and their support is gratefully acknowledged.

\section{Boundary Regularity For the Ricci EQuation}

In this section we describe the key results on local regularity at the boundary of a metric tensor on which there are Ricci curvature bounds and a Lipschitz bound on the mean curvature. Our set-up is the following.

Let $\mathcal{B}$ be a ball about $0 \in \mathbb{R}^{n}, \Omega=\mathcal{B} \cap\left\{x: x^{n}>0\right\}$. Let $\Sigma=\mathcal{B} \cap\left\{x: x^{n}=0\right\}$ and set $\bar{\Omega}=\Omega \cup \Sigma$. Let $g$ be a metric tensor on $\bar{\Omega}$, and denote by $h$ its restriction 
to $\Sigma$. We make the following hypotheses:

$$
\begin{aligned}
g_{j k} & \in H^{1, p}(\Omega), \quad \text { for some } p>n, \\
h_{j k} & \in H^{1,2}(\Sigma), \quad 1 \leq j, k \leq n-1, \\
\operatorname{Ric}^{\Omega} & \in L^{\infty}(\Omega), \\
\operatorname{Ric}^{\Sigma} & \in L^{\infty}(\Sigma), \\
H & \in \operatorname{Lip}(\Sigma) .
\end{aligned}
$$

Here $H$ denotes the mean curvature of $\Sigma \subset \bar{\Omega}$, i.e., $H=\operatorname{Tr} A /(n-1)$, where $A$ is the Weingarten map, a section of $\operatorname{End}(T \Sigma)$. Our goal is to establish the following result.

Theorem 2.1. Under the hypotheses (2.1)-(2.5), given $z \in \bar{\Omega}$, there exist local harmonic coordinates on a neighborhood $\bar{U}$ of $z$ in $\bar{\Omega}$ with respect to which

$$
g_{j k} \in C_{*}^{2}(\bar{U}) .
$$

Here $C_{*}^{2}(\bar{U})$ is a Zygmund space, as mentioned in $\S 1$. The harmonic coordinates for which (2.6) holds are arbitrary coordinates $\left(u^{1}, \ldots, u^{n}\right)$ satisfying $\Delta u^{j}=0$ on a chart not intersecting $\Sigma$. On a neighborhood of a point in $\Sigma$, these coordinates are "boundary harmonic coordinates," which are defined as follows. We require $\left(u^{1}, \ldots, u^{n}\right)$ to be defined and regular of class at least $C^{1}$ on a neighborhood of $z$ in $\bar{\Omega}$, and $\Delta u^{j}=0$. We require that $v^{j}=\left.u^{j}\right|_{\Sigma}$ be harmonic on $\Sigma$, i.e., annihilated by the Laplace-Beltrami operator of $\Sigma$ with its induced metric tensor. We require that $u^{n}$ vanish on $\Sigma$, and that $\left(u^{1}, \ldots, u^{n}\right)$ map a neighborhood of $z$ in $\bar{\Omega}$ diffeomorphically onto $\bar{\Omega}$.

Regarding the fact that the hypotheses (2.1)-(2.2) imply that various curvature tensors are well defined, it can be shown that

$$
\begin{aligned}
g_{j k} \in C(\bar{\Omega}) \cap H^{1,2}(\Omega) & \Longrightarrow \Gamma \in L^{2}(\Omega), R_{b j k}^{a} \in H^{-1,2}(\Omega)+L^{1}(\Omega) \\
& \Longrightarrow \operatorname{Ric}_{b k} \in H^{-1,2}(\Omega)+L^{1}(\Omega) .
\end{aligned}
$$

The hypothesis (2.1) is stronger than the hypothesis in (2.7). It implies $g_{j k} \in C^{r}(\bar{\Omega})$ for some $r>0$, so (2.7) is applicable both to $g_{j k}$ on $\Omega$ and, in view of (2.2), to $h_{j k}$ on $\Sigma$. One also shows that the Weingarten map has the property $A \in B_{p, p}^{-1 / p}(\Sigma)$, as a consequence of (2.1). Thus we have a priori that $H \in B_{p, p}^{-1 / p}(\Sigma)$, and the hypothesis (2.5) strengthens this condition on $H$, in a fashion that is natural for the desired conclusion of Theorem 2.1.

Our approach to the proof of Theorem 2.1 is to obtain the result as a regularity result for an elliptic boundary problem. We use the PDE (1.1) (the "Ricci equation") for the components of the metric tensor, in boundary harmonic coordinates, and use Dirichlet boundary conditions on some components of $g_{j k}$ and Neumann boundary conditions on complementary components; see (2.9) and (2.12)-(2.13) for a more precise description.

The proof of Theorem 2.1 in [AK2LT] is done in stages. First it is shown that the conclusion of Theorem 2.1 holds when the hypotheses (2.1)-(2.2) are strengthened a bit. 
Proposition 2.2. In the setting of Theorem 2.1, replace hypotheses (2.1)-(2.2) by

$$
g_{j k} \in C^{1+s}(\bar{\Omega}) \text {, for some } s \in(0,1) \text {, }
$$

and retain hypotheses (2.3)-(2.5). Then the conclusion (2.6) holds.

In addition to providing a first step toward establishing Theorem 2.1, Proposition 2.2 is itself sufficiently strong for the application to geometric convergence described in $\S 3$.

The demonstration of Proposition 2.2 begins with a construction of boundary harmonic coordinates, mentioned above. In these new coordinates, (2.8) and (2.3)(2.5) are preserved. Now in harmonic coordinates the metric tensor satisfies the elliptic PDE (1.1), and from (2.8) and (2.3) we have $F_{\ell m}=B_{\ell m}-2 \operatorname{Ric}_{\ell m} \in L^{\infty}(\Omega)$, and the coefficients of $\Delta$ have the same degree of regularity as $g_{j k}$ in (2.8).

Now, if $j, k \leq n-1$, then well known local regularity results on $\Sigma$ following from (2.4) give $\left.g_{j k}\right|_{\Sigma}=h_{j k} \in H^{2, p}(\Sigma), \forall p<\infty$, but in fact there is the following refinement, established in Proposition III.10.2 of [T2]:

$$
\left.g_{j k}\right|_{\Sigma}=h_{j k} \in \mathfrak{h}^{2, \infty}, \quad 1 \leq j, k \leq n-1 .
$$

Here $\mathfrak{h}^{2, \infty}$ denotes the bmo-Sobolev space of functions whose derivatives of order $\leq 2$ belong to bmo, the localized space of functions of bounded mean oscillation. We have the following (after perhaps shrinking $\bar{\Omega}$ to a smaller neighborhood of $z$ ). See AK2LT for details.

Lemma 2.3. Under the hypotheses of Proposition 2.2, we have, in boundary harmonic coordinates,

$$
g_{j k} \in C_{*}^{2}(\bar{\Omega}), \quad 1 \leq j, k \leq n-1 .
$$

To continue, following [An2], we switch over to PDE for $g^{\ell m}$. Parallel to (1.1), we have

$$
\Delta g^{\ell m}=B^{\ell m}(g, \nabla g)+2\left(\operatorname{Ric}^{\Omega}\right)^{\ell m}=F^{\ell m},
$$

and (2.8) and (2.3) give $F^{\ell m} \in L^{\infty}(\Omega)$. We take $m=n$ and proceed to derive Neumann-type boundary conditions for the components $g^{\ell n}, 1 \leq \ell \leq n$. In fact, as shown in AK2LT,

$$
N g^{n n}=-2(n-1) H g^{n n}, \quad \text { on } \Sigma,
$$

and, for $1 \leq \ell \leq n-1$,

$$
N g^{\ell n}=-(n-1) H g^{\ell n}+\frac{1}{2} \frac{1}{\sqrt{g^{n n}}} g^{\ell k} \partial_{k} g^{n n}, \quad \text { on } \Sigma .
$$

Here $H$ is the mean curvature of $\Sigma$, which we assume satisfies (2.5), and $N$ is the unit normal field to $\Sigma$, pointing inside $\Omega$.

Having (2.11)-(2.13), we can establish further regularity of the functions $g^{\ell n}$. The following is proven in AK2LT.

Lemma 2.4. In boundary harmonic coordinates, we have

$$
g^{\ell n} \in C_{*}^{2}(\bar{\Omega}), \quad 1 \leq \ell \leq n .
$$

One then verifies that Lemmas 2.3 and 2.4 yield $g_{n \ell}=g_{\ell n} \in C_{*}^{2}(\bar{\Omega})$. These results yield Proposition 2.2. To establish Theorem 2.1 in full strength, we need to work harder, especially on the Neumann problem. We continue to have (2.11); however, this time it is not so straightforward to produce the Neumann-type boundary 
conditions (2.12)-(2.13). Consider (2.12). The right side is well defined; we have $\left.H g^{n n}\right|_{\Sigma} \in C^{s}(\Sigma)$, for some $s>0$. As for $N$, the unit normal field to $\Sigma$ is also Hölder continuous of class $C^{r}$. But applying $N$ to $g^{n n} \in H^{1, p}(\Omega)$ does not yield an object that can be evaluated on $\Sigma$. One has the same problem with the left side of (2.13), and the right side of (2.13) is also problematic.

However, we are able to show that a weak formulation of the Neumann boundary condition is applicable, and we establish regularity results for weak solutions to the Neumann problem strong enough to complete the proof of Theorem 2.1. See AK2LT for details.

\section{Geometric CONVERGEnCE FOR MANifolds With BOUNDARY}

A sequence $\left(\bar{M}_{k}, g_{k}\right)$ of compact Riemannian manifolds with boundary $\partial M_{k}$ is said to converge in the $C^{r}$-topology (given $0<r<\infty$ ) to a compact Riemannian manifold $(\bar{M}, g)$ provided that $g$ is a $C^{r}$ metric tensor on $\bar{M}$ and, for $k$ sufficiently large, there exist diffeomorphisms $F_{k}: \bar{M} \rightarrow \bar{M}_{k}$ such that $F_{k}^{*} g_{k}$ converges to $g$ in the $C^{r}$-topology. (Necessarily $F_{k}: \partial M \rightarrow \partial M_{k}$.) In this section we will identify classes of Riemannian manifolds with boundary that are pre-compact in the $C^{r}$-topology, for any given $r<2$.

We work with families of Riemannian manifolds with boundary of the following sort. Fix the dimension, $n$. Given $R_{0}, i_{0}, S_{0}, d_{0} \in(0, \infty)$, denote by $\mathcal{M}\left(R_{0}, i_{0}, S_{0}, d_{0}\right)$ the class of compact, connected, $n$-dimensional Riemannian manifolds with boundary $(\bar{M}, g)$, with smooth metric tensor, with the following four properties:

$$
\left\|\operatorname{Ric}_{M}\right\|_{L^{\infty}(M)} \leq R_{0}, \quad\left\|\operatorname{Ric}_{\partial M}\right\|_{L^{\infty}(\partial M)} \leq R_{0},
$$

where Ric denotes the Ricci tensor.

$$
i_{M} \geq i_{0}, \quad i_{\partial M} \geq i_{0}, \quad i_{b} \geq 2 i_{0} .
$$

Here $i_{M}$ denotes the injectivity radius of $\bar{M}, i_{\partial M}$ that of $\partial M$, and $i_{b}$ the boundary injectivity radius of $\bar{M}$.

$$
\|H\|_{\operatorname{Lip}(\partial M)} \leq S_{0},
$$

where $H$ is the mean curvature of $\partial M$ in $\bar{M}$.

$$
\operatorname{diam}(\bar{M}, g) \leq d_{0} .
$$

We recall the concept of boundary injectivity radius, $i_{b}$. It is the optimal quantity with the following property. Namely, there is a collar neighborhood $\mathcal{C}$ of $\partial M$ in $\bar{M}$ and a (unique) proper function $f \in C^{2}(\mathcal{C})$ such that $\left.f\right|_{\partial M}=0,|\nabla f| \equiv 1$, $f(\mathcal{C}) \supset\left[0, i_{b}\right)$. With this, local coordinates $\left(v^{1}, \ldots, v^{n-1}\right)$ on an open set in $\partial M$ can be continued inside, as constant on the integral curves of $\nabla f$, to produce, along with $v^{n}=f$, a set of "boundary normal coordinates." To further clarify the first part of (3.2), we mean that $\operatorname{Exp}_{p}: B_{\rho}(0) \rightarrow M$, where $B_{\rho}(0)=\left\{v \in T_{p} M: g(v, v)<\rho^{2}\right\}$, is a diffeomorphism for $\rho=i_{0}$ if $\operatorname{dist}(p, \partial M) \geq i_{0}$, and it is a diffeomorphism for $\rho=\operatorname{dist}(p, \partial M)$ if $\operatorname{dist}(p, \partial M) \leq i_{0}$.

The main result announced in this section is the following.

Theorem 3.1. Given $R_{0}, i_{0}, S_{0}, d_{0} \in(0, \infty), \mathcal{M}\left(R_{0}, i_{0}, S_{0}, d_{0}\right)$ is precompact in the $C^{r}$-topology for each $r<2$. In particular, any sequence $\left(\bar{M}_{k}, g_{k}\right)$ in $\mathcal{M}\left(R_{0}, i_{0}, S_{0}, d_{0}\right)$ has a subsequence that converges in the $C^{r}$-topology to a limit $(\bar{M}, g)$. Furthermore, the metric tensor $g$ belongs to $C_{*}^{2}(\bar{M})$. 
Such a result was established in [An1] in the category of compact manifolds without boundary; subsequently there have been expositions in $[\mathrm{HH}]$ and in $[\mathrm{Pe}]$. Our proof of Theorem 3.1 follows the structure of the argument in [An1, with necessary modifications to treat the case of nonempty boundary. In this regard the boundary regularity results of $\S 2$ play a major role. The $C_{*}^{2}$ part of the conclusion is also more precise than that noted in earlier results. This precision will be of major value in the application of Theorem 3.1 to results on inverse boundary spectral problems presented in $\S 4$.

One ingredient in the proof of Theorem 3.1 is to demonstrate the applicability of a well known "abstract" convergence result, of the sort given in [Pe, pp. 293-296 (extended in the natural fashion to manifolds with boundary). To show that this applies, it suffices to construct sufficiently good boundary harmonic coordinates. Doing this brings in the notion of $C^{s}$-harmonic radius, introduced in An1 in the context of manifolds without boundary. Roughly speaking, there are boundary harmonic coordinates on balls of this radius, in which the metric tensor satisfies uniform $C^{s}$ bounds. Similarly there is a natural notion of $C_{*}^{2}$-harmonic radius. We denote the $C_{*}^{2}$-harmonic radius of $(\bar{M}, g)$ by

$$
r_{h}(\bar{M}, g, Q),
$$

where $Q$ quantifies these bounds on the metric tensor.

The goal then becomes to show that, given $R_{0}, i_{0}, S_{0}, d_{0} \in(0, \infty)$, there is a lower bound on the $C_{*}^{2}$-harmonic radius of $(\bar{M}, g) \in \mathcal{M}\left(R_{0}, i_{0}, S_{0}, d_{0}\right)$. The following key result is proven in $\mathrm{AK} 2 \mathrm{LT}$.

Theorem 3.2. Let $R_{0}, i_{0}, S_{0}$, and $d_{0}$ be given, in $(0, \infty)$, and let $Q \in(1,2)$ be given. Then there exists $r_{\mathcal{M}}=r_{\mathcal{M}}\left(R_{0}, i_{0}, S_{0}, d_{0}, Q\right)>0$ such that

$$
r_{h}(\bar{M}, g, Q) \geq r_{\mathcal{M}}, \quad \forall(\bar{M}, g) \in \mathcal{M}\left(R_{0}, i_{0}, S_{0}, d_{0}\right) .
$$

The proof of Theorem 3.2 given in [AK2LT] involves the following ingredients: a blow-up argument, use of the regularity results of $\S 2$ and of the fundamental equations of hypersurface theory, and use of the Cheeger-Gromoll splitting theorem.

Remark. Invoking the definition of the Gromov-Hausdorff topology (cf. $\mathrm{Gr}$ ) we can show that $\overline{\mathcal{M}\left(R_{0}, i_{0}, S_{0}, d_{0}\right)}$ is compact in the Gromov-Hausdorff topology and $C^{r}$-convergence is equivalent to Gromov-Hausdorff convergence on this compact set, for any $r \in[1,2)$.

\section{Gel'FAND INVERSE BOUNDARY PROBLEM}

In this section we discuss uniqueness and stability for the inverse boundary spectral problem. To fix notations, assume that $(\bar{M}, g, \partial M)$ is a compact, connected manifold, with nonempty boundary, provided with a metric tensor $g$ with some limited smoothness (specified more precisely below). Let $\Delta^{N}$ be the Neumann Laplacian. Denote by $\left(\lambda_{k}\right)_{k=0}^{\infty}$ its eigenvalues (counting multiplicity) and by $\left(\phi_{k}\right)_{k=0}^{\infty}$ the corresponding eigenfunctions.

The Gel'fand inverse boundary problem (in its spectral formulation) is the problem of the reconstruction of $(\bar{M}, g)$ from its boundary spectral data, i.e., the collection $\left(\partial M,\left\{\lambda_{k},\left.\phi_{k}\right|_{\partial M}\right\}_{k=1}^{\infty}\right)$. (We have mentioned Gel'fand's original formulation in the Introduction.)

The following uniqueness result is proven in [AK2LT. 
Theorem 4.1. Let $\bar{M}$ be a compact, connected manifold with nonempty boundary and $C_{*}^{2}$ metric tensor. Then the boundary spectral data $\left(\partial M,\left\{\lambda_{k},\left.\phi_{k}\right|_{\partial M}\right\}_{k=1}^{\infty}\right)$ determine the manifold $\bar{M}$ and its metric $g$ uniquely.

Such a result was established in the $C^{\infty}$ case in [BK1]. Some different techniques are required to treat the $C_{*}^{2}$ case. We briefly describe how to determine $\bar{M}$ as a topological space, referring to [AK2LT] for further details.

We start with the introduction of some useful geometric objects. Let $\Gamma \subset \partial M$ be open and take $t \geq 0$. Then we set

$$
M(\Gamma, t)=\{x \in M: d(x, \Gamma) \leq t\},
$$

the domain of influence of $\Gamma$ at "time" $t$, and define

$$
\mathbf{L}(\Gamma, t)=\mathcal{F} L^{2}(M(\Gamma, t)) \subset \ell^{2} .
$$

Here, $\mathcal{F}$ stands for the Fourier transform of functions from $L^{2}(M)$, i.e., $\mathcal{F}(u)=$ $\left\{u_{k}\right\}_{k=1}^{\infty} \in \ell^{2}, u_{k}=\left(u, \phi_{k}\right)_{L^{2}(M)}$, and the subspace $L^{2}(M(\Gamma, t))$ consists of all functions in $L^{2}(M)$ with support in the set $M(\Gamma, t)$.

One ingredient in the proof of Theorem 4.1 is the following. Consider the wave equation

$$
\begin{aligned}
& \left(\partial_{t}^{2}-\Delta\right) u^{f}(x, t)=0 \quad \text { in } M \times \mathbb{R}_{+}, \\
& \left.u^{f}\right|_{t=0}=0,\left.\quad u_{t}^{f}\right|_{t=0}=0,\left.\quad N u^{f}\right|_{\partial M \times \mathbb{R}_{+}}=f \in C_{0}^{1}(\Gamma \times(0, T)),
\end{aligned}
$$

where $N$ is the exterior unit normal field to $\partial M$. Using Tataru's unique continuation theorem [Ta], it was shown in [Be1] (also Theorem 3.10 of [KKL]) that the following holds.

Proposition 4.2. For each $T>0$, the set $\left\{u^{f}(T): f \in L^{2}(\Gamma \times(0, T))\right\}$ is a dense subspace of $L^{2}(M(\Gamma, T))$.

Meanwhile, the following formula (due to Blagoveshchenskii) gives the Fourier coefficients $u_{k}^{f}(t)$ of a wave $u^{f}(\cdot, t)$ in terms of the boundary spectral data,

$$
u_{k}^{f}(t)=\int_{0}^{t} \int_{\partial M} f\left(x, t^{\prime}\right) \frac{\sin \sqrt{\lambda_{k}}\left(t-t^{\prime}\right)}{\sqrt{\lambda_{k}}} \phi_{k}(x) d S_{g} d t^{\prime} .
$$

This, together with Proposition 4.2, implies the following.

Corollary 4.3. Given $\Gamma \subset \partial M$ and $t>0$, the boundary spectral data determine the subspace

$$
\mathbf{L}(\Gamma, t)=\mathcal{F} L^{2}(M(\Gamma, t)) \subset \ell^{2} .
$$

Now let $h \in C(\partial M)$. We can ask if $h$ is the boundary distance function for some $x \in M$. In fact, use of Corollary 4.3 allows one to answer this question. It follows that the boundary spectral data determine the image in $L^{\infty}(\partial M)$ of the boundary distance representation $R$. Here, $R: \bar{M} \rightarrow C(\partial M)$ is defined by

$$
R(x)=r_{x}(\cdot), \quad r_{x}(z)=\operatorname{dist}(x, z), \quad z \in \partial M .
$$

(Compare $[\mathrm{KKL}]$ and $[\mathrm{Ku}]$.) Clearly, the map $R$ is Lipschitz continuous. Moreover, under the assumptions of Theorem 4.1 it is injective. This follows from Osgood's theorem applied to geodesics normal to $\partial M$.

Since $\bar{M}$ is compact, injectivity and continuity imply that $R$ is a homeomorphism, i.e., $R(\bar{M})$ with the distance inherited from $L^{\infty}(\partial M)$ and $(\bar{M}, g)$ are homeomorphic, 
and thus $R(\bar{M})$ can be identified with $\bar{M}$ as a topological manifold. We hence have the following.

Proposition 4.4. Assume $\left(\bar{M}_{1}, g_{1}\right)$ and $\left(\bar{M}_{2}, g_{2}\right)$ satisfy the hypotheses of Theorem 4.1. If they have identical boundary spectral data, including $\partial M_{1}=\partial M_{2}=X$, as $C^{2}$ manifolds, then there is a natural correspondence of $R\left(\bar{M}_{1}\right)$ and $R\left(\bar{M}_{2}\right) \subset$ $C(X)$, producing a uniquely defined homeomorphism

$$
\chi: \bar{M}_{1} \longrightarrow \bar{M}_{2} \text {. }
$$

We refer to $[\mathrm{AK} 2 \mathrm{LT}]$ for a demonstration that $\chi$ in (4.7) is a $C^{2}$-diffeomorphism, preserving the metric tensors, which proves the uniqueness result of Theorem 4.1.

We next consider stabilization of inverse problems using geometric convergence results and apply them to the Gel'fand problem. The basic thrust of our argument provides an illustration of a general "stabilization principle for inverse problems."

Let us set up some notation. Denote by $\mathcal{M}_{X}\left(C_{*}^{2}\right)$ the set of compact, connected manifolds $\bar{M}$ with nonempty boundary $X$, endowed with a metric tensor in $C_{*}^{2}(\bar{M})$. Given $(\bar{M}, g) \in \mathcal{M}_{X}\left(C_{*}^{2}\right)$, set

$$
\mathcal{D}(\bar{M}, g)=\left\{\lambda_{j},\left.\phi_{j}\right|_{X}\right\}_{j=1}^{\infty},
$$

the right side denoting the boundary spectral data of $(\bar{M}, g)$. We have

$$
\mathcal{D}: \mathcal{M}_{X}\left(C_{*}^{2}\right) \longrightarrow \mathcal{B}_{X}
$$

where $\mathcal{B}_{X}$ denotes the set of sequences $\left\{\mu_{j}, \psi_{j}: j \geq 1\right\}$, with $\mu_{j} \in \mathbb{R}^{+}, \mu_{j} \nearrow+\infty$, and $\psi_{j} \in L^{2}(X)$, modulo an equivalence relation, which can be described as follows. We say $\left\{\mu_{j}, \psi_{j}\right\} \sim\left\{\mu_{j}, \tilde{\psi}_{j}\right\}$ if $\psi_{j}(x)=\alpha_{j} \tilde{\psi}_{j}(x)$ for some $\alpha_{j} \in \mathbb{C},\left|\alpha_{j}\right|=1$. More generally, if $\mu_{k_{0}}=\cdots=\mu_{k_{1}}$, we allow

$$
\psi_{j}(x)=\sum_{k=k_{0}}^{k_{1}} \alpha_{j k} \tilde{\psi}_{k}(x), \quad j=k_{0}, \ldots, k_{1},
$$

for a unitary $l \times l$ matrix $\left(\alpha_{j k}\right), l=k_{1}-k_{0}+1$. The content of Theorem 4.1 is that the map (4.9) is one-to-one.

There are natural topologies one can put on the sets in (4.9). Furthermore, it follows from standard techniques of perturbation theory (cf. $[\mathrm{K}]$ ) that $\mathcal{D}$ is continuous in (4.9).

Now the map (4.9) is by no means invertible, giving rise to the phenomenon of ill-posedness. One wants to "stabilize" the inverse problem, showing that certain a priori hypotheses on the domain $(\bar{M}, g)$ put it in a subset $K \subset \mathcal{M}_{X}\left(C_{*}^{2}\right)$ with $\mathcal{D}^{-1}$ acting continuously on the image of $K$. The results of $\S 3$ provide a tool to accomplish this.

Recall the class $\mathcal{M}\left(R_{0}, i_{0}, S_{0}, d_{0}\right)$ defined in $\S 3$. Given a boundary $X$, denote by $\mathcal{M}_{X}\left(R_{0}, i_{0}, S_{0}, d_{0}\right)$ the set of such manifolds with boundary $X$. It follows from Theorem 3.1 that $\overline{\mathcal{M}_{X}\left(R_{0}, i_{0}, S_{0}, d_{0}\right)}$ is compact in the $C^{r}$ topology, for any $r \in$ $(1,2)$, and is contained in $\mathcal{M}_{X}\left(C_{*}^{2}\right)$. We hence give $\overline{\mathcal{M}_{X}\left(R_{0}, i_{0}, S_{0}, d_{0}\right)}$ the $C^{r}$ topology, and we see this is independent of $r$, for $r \in(1,2)$.

Combined with Theorem 4.1, these observations yield the following conditional stability of the Gel'fand inverse problem. 
Theorem 4.5. Given $R_{0}, i_{0}, S_{0}, d_{0} \in(0, \infty)$,

$$
\mathcal{D}: \overline{\mathcal{M}_{X}\left(R_{0}, i_{0}, S_{0}, d_{0}\right)} \longrightarrow \mathcal{B}_{X}
$$

is a homeomorphism of $\overline{\mathcal{M}_{X}\left(R_{0}, i_{0}, S_{0}, d_{0}\right)}$ onto its range, $\mathcal{B}_{X}\left(R_{0}, i_{0}, S_{0}, d_{0}\right)$; hence

$$
\mathcal{D}^{-1}: \mathcal{B}_{X}\left(R_{0}, i_{0}, S_{0}, d_{0}\right) \longrightarrow \overline{\mathcal{M}_{X}\left(R_{0}, i_{0}, S_{0}, d_{0}\right)}
$$

is continuous.

\section{REFERENCES}

[An1] M. Anderson, Convergence and rigidity of manifolds under Ricci curvature bounds, Invent. Math. 102 (1990), 429-445. MR 92c:53024

[An2] M. Anderson, Boundary regularity, uniqueness, and non-uniqueness for AH Einstein metrics on 4-manifolds, Advances in Math., to appear.

[AK2LT] M. Anderson, A. Katsuda, Y. Kurylev, M. Lassas, and M. Taylor, Boundary regularity for the Ricci equation, geometric convergence, and Gel'fand's inverse boundary problem, Preprint, 2002.

[Be1] M. Belishev, An approach to multidimensional inverse problems, Dokl. Akad. Nauk. SSSR 297 (1987), 524-527; English transl., Soviet Math. Dokl. 36 (1988), no. 3, 481484. MR 89c:35152

[BK1] M. Belishev and Y. Kurylev, To the reconstruction of a Riemannian manifold via its spectral data (BC-method), Comm. PDE 17 (1992), 767-804. MR 94a:58199

[Ch] J. Cheeger, Finiteness theorems for Riemannian manifolds, Amer. J. Math. 92 (1970), 61-75. MR 41:7697

[CG] J. Cheeger and D. Gromoll, The splitting theorem for manifolds with non-negative Ricci curvature, J. Diff. Geom. 6 (1971), 119-128. MR 46:2597

[DTK] D. De Turck and J. Kazdan, Some regularity theorems in Riemannian geometry, Ann. Scient. Ecole Norm. Sup. Paris 14 (1981), 249-260. MR 83f:53018

[Ge] I. Gel'fand, Some aspects of functional analysis and algebra, Proc. ICM 1 (1954), 253277. MR 20:1925

[Gr] M. Gromov, Metric Structures for Riemannian and Non-Riemannian Spaces (with appendices by M. Katz, P. Pansu, and S. Semmes), Birkhäuser, Boston, 1999. MR 2000d:53065

[HH] M. Hebey and M. Herzlich, Harmonic coordinates, harmonic radius, and convergence of Riemannian manifolds, Rend. di Mathem. Ser. VII, 17 (1997), 569-605. MR 99f:53039

[KKL] A. Katchalov, Y. Kurylev, and M. Lassas, Inverse Boundary Spectral Problems, Chapman-Hall/CRC Press, Boca Raton, 2001. MR 2003e:58045

[K] T. Kato, Perturbation Theory for Linear Operators, Springer-Verlag, New York, 1966. MR 34:3324

[Ka] A. Katsuda, BC-method and stability of Gel'fand inverse spectral problem, Proc. Conf. "Spectral and Scattering Theory," RIMS, Kyoto, 2001, 24-35.

[K2L] A. Katsuda, Y. Kurylev, and M. Lassas, Stability in Gelfand inverse boundary spectral problem, Preprint, 2001.

[Ku] Y. Kurylev, Multidimensional Gel'fand inverse boundary problem and boundary distance map. In: Inverse Problems Related to Geometry (H. Soga, ed.), 1-15, Ibaraki Univ. Press, Mito, 1997.

$[\mathrm{KuL}] \quad$ Y. Kurylev and M. Lassas, The multidimensional Gel'fand inverse problem for non-selfadjoint operators, Inverse Problems 13 (1997), 1495-1501. MR 98m:35224

[Pe] P. Petersen, Riemannian Geometry, Springer-Verlag, New York, 1998. MR 98m:53001 
GEOMETRIC REGULARITY AND CONVERGENCE, AND INVERSE PROBLEMS

[Ta] D. Tataru, Unique continuation for solutions to PDE's: between Hörmander's theorem and Holmgren's theorem, Comm. PDE 20 (1995), 855-884. MR 96e:35019

[T2] M. Taylor, Tools for PDE, AMS, Providence, RI, 2000. MR 2001g:35004

Mathematics Department, State University of New York, Stony Brook, Ny 11794

E-mail address: anderson@math.sunysb.edu

Mathematics Department, Okayama University, Tsushima-naka, Okayama, 700-8530, JAPAN

E-mail address: katsuda@math.okayama-u.ac.jp

Department of Mathematical Sciences, Loughborough University, Loughborough, LE11 3TU, UK

E-mail address: Y.V.Kurylev@lboro.ac.uk

Rolf Nevanlinna Institute, University of Helsinki, Fin-00014, Finland

E-mail address: lassas@cc.helsinki.fi

Mathematics Deptartment, University of North Carolina, Chapel Hill, NC 27599

E-mail address: met@math.unc.edu 